\title{
3 则奇莳毛发医学病例及其分析
}

\author{
Analysis of Three Cases of Exotic Hair Medicine \\ 刘作华 ${ }^{1 a}$ 朱森 $^{1 \mathrm{~b}}$ 庞张宇 ${ }^{1 \mathrm{c}}$ 杜强 $^{2}$ 张勇 $^{1 \mathrm{a}}$ \\ Zuohua Liu ${ }^{\text {la }}$ Miao Zhu ${ }^{\text {lb }}$ Zhangyu Pang ${ }^{\text {lc }}$ Qiang Du ${ }^{2}$ Yong Zhang ${ }^{\text {la }}$
}

\author{
1.川北医学院 a.l临床医学系; b. 护理学院; \\ c.医学影像技术系 \\ 中国・四川 南充 637000 \\ 2.西华大学土木建筑与环境学院 \\ 中国・四川成都 610000 \\ 1. North Sichuan Medical College a. Department of \\ Clinical Medicine; $b$. School of Nursing; \\ c. Department of Medical Imaging Technology, \\ Nanchong, Sichuan, 637000, China \\ 2. School of Civil Architecture and Environment, \\ Xihua University, \\ Chengdu, Sichuan, 610000, China
}

【摘要】毛发本来就毫不起眼, 除头发外, 人体其他地方有关的毛发疾病(如睫毛疾病) 也 鲜为人知, 有关毛发的奇范医学病例更是少之又少。毛发长在合适的地方就会起保护作用, 而不该长出毛发的地方 (如论文提到的眼内) 长出就会导致疾病。论文搜集了 3 则与人体毛 发有关的奇范医学病例临床特点及发病因素并展开分析, 旨在为临床提供指导。

【Abstract】In addition to hair, hair is rarely known for hair disorders such as eyelash diseases in other parts of the body, and rare medical cases of hair are rare. Hair that grows in the right place protects it, and where it shouldn't (as mentioned in this article) it causes disease. The clinical features and pathogenesis of three exotic medical cases related to human hair were analyzed in order to provide clinical guidance.

【关键词】毛发;寄生虫病; 皮毛迁移; 角膜缘皮肤病; 奇㴰病例

【Keywords】hair; parasitic diseases; fur migration; limbic keratopathy; exotic cases

【DOI】10.36012/pmr.v2i1.1319

\section{1 一般资料}

案例一:跳着约 200 条寄生虫的睫毛

患者小林,女,20 岁,居住于南京农村,三个月前突发眼睛 瘁痒,病因不明,未去医院就诊, 自行购买止痒药物, 药名不 详,揉搓眼睛后痹痒消除。一天前因眼睛红肿发痒遂到南京中 医院眼科门诊部就医, 自述有宠物狗接触史, 饮食、睡眠正常, 体重正常, 否认眼部外伤史, 否认糖尿病高血压, 否认传染病 史、遗传病史,无过敏史。医生视诊时意外发现小林的睫毛上 跳满了寄生虫, 几乎每根睫毛根部都有一条寄生虫紧紧抱着 睫毛, 寄生虫数估算有 200 条。遂主治医生用消毒棉签尝试擦 拭掉寄生虫, 无果后行眼睑睫毛齐根剪切术 ${ }^{[1]}$ 。南京中医院眼 科副主任医师刘昳根据这些寄生虫的外形和习性, 初步判断 是狗䖝感染, 后经检验科证实寄生虫确为䖝。经小林验证, 自 己特别喜欢狗, 所以家里养了一只狗, 并且跟狗密切接触, 就 连晚上也睡在一起。

\section{案例二:眼睛里面长出毛}

患者男,19 岁,两年前突发眼睛轻度瘙痒,遂去当地医院 眼科就诊, 行眼底镜检查后未发现异常, 给予止痒药物治疗, 药名不详, 服用后瘙痒减退。一天前因视力模糊来到我院眼科 就诊, 患者自述无疼痛, 但肿块造成视力缺陷, 眨眼时有轻微 的不适, 并且间歇有异物存在的感觉, 饮食、睡眠正常, 体重正 常, 否认眼部外伤史, 否认糖尿病高血压, 否认传染病史、遗传 病史, 无过敏史。遂行眼底镜检查, 发现右眼有一个白色的卵 圆形肿块, 直径为 $5 \mathrm{~mm} \times 6 \mathrm{~mm}$, 横跨㑔下缘, 肿块处长出几根黑 毛。视力检查: 左眼视力为 $20 / 20$, 右眼视力为 $20 / 60$, 右眼有弱 视和散光, 眼压正常 ${ }^{[2]}$ 。后组织病理证实为角膜缘皮肤病。遂进 行手术切除, 由于美容原因行板层角膜移植术, 术后弱视和散 光无明显改善。

案例三:皮肤毛迁移(CPM)

患者男, 6 个月大, 12 小时前他的母亲注意到贞儿左腿后 侧有一条黑色的、螺纹状的线条,并且观察到这条线在婴儿的 
腿上移动,未给予治疗。当天因婴儿左腿后侧出现大量皮疹于 我院皮肤科就诊, 发病前期贞儿没有出现瘙痒, 饮食、睡眠不 良, 体重降低, 否认眼部外伤史, 否认糖尿病高血压, 否认传染 病史、遗传病史,无过敏史, CPM 的实际提交期不详。体格检查 发现左腿后侧有一个表皮破裂，在皮肤表面清晰地观察到一 条细小的、非常浅薄的黑线,病灶周围无炎症征象。遂用外科 小钳子从破裂处拔出一根棕色的头发, 长 2.5 厘米, 从表皮床 上拔出, 少量出血 ${ }^{[3]}$ 。黑线显示为无毛囊的裸发轴, 经验证病人 的头发轴与他母亲的头发非常相似, 进一步说明疾病可能是 母亲常年抱孩子，母亲头发有更多的机会接触到婴儿的皮肤 而引起的。去除毛发后, 皮疹立即消退。

\section{2 讨论}

案例一: 䖝子的成虫和若虫终生在宿主身上吸血, 宿主主 要是陆生哺乳动物, 通过接触传播。䖝子不仅吸血, 而且会使 宿主奇痒不止, 并能传染给很多人畜共患病。狗为家庭中最常 养的宠物之一, 䖝子常附着在狗的毛发上, 而狗又有称舐毛发 的习性。狗㖨毛时舌头上黏上䖝子, 再称舐主人面部时, 闽子 也发生了转移。禹子有群集的习性, 头䖝主要寄生在头部毛发 中, 多集中在耳后发根, 体䖝则多集中于衣领、衣缝、裤腰处。 眼睛是个湿润干净、有毛发生长的部位, 容易成为寄生虫的聚 集地。寄生虫长期寄居在人的眼睛里, 容易引发结膜炎等眼部 炎症。䖝子还能传播回归热等世界传染性疾病, 回归热螺旋体 寄生于䖝子, 当闽子咬人时, 被咬部位很痒, 人在用力抓痒时, 会把闽子挤破，它体液内的病原体会随抓痒而带入被咬的伤 口引起疾病 ${ }^{[4-5]}$ 。因此, 饲养宠物要格外注意与其保持距离, 定 期给宠物驱虫、清洁, 常用热水肥皇洗澡, 并时常换衣服, 注意 环境卫生。一旦发生眼部或身体其他部位不适, 应尽早就诊。

案例二: 鉴别诊断包括角膜缘皮样、异物肉芽肿、不典型 翼状怒肉和角膜瘢痕。不典型翼状胬肉一般认为是受风尘、日 光、烟雾等长期的慢性外界刺激而引起的一种慢性炎症性病 变，单眼或双眼受犯，为睑裂部球结膜及结膜下组织发生变 性、肥厚、增生,向角膜内发展, 呈三角形的一种赘生组织, 侵 犯角膜后日渐增大, 甚至可覆盖至瞳孔区而严重影响视力, 但 赘生物中无毛发等新生物。异物肉芽肿是非机体组织的各种
物质(如非金属碎片、玻璃、化纤、寄生性幼虫等)进人人体后, 在皮肤组织引起的过敏反应, 其结构为肉芽肿性炎症反应。通 常异物进入机体到皮疹出现的时间较长, 可由数月到数年不 等。组织病理检测巨噬细胞及异物巨细胞包围异物或出现结 核性结节, 再结合临床表现和皮损特点即可鉴别。角膜缘皮样 的鉴别标志主要是肿块的形状和其内新生物, 新生物可以是 毛发、牙齿等。

案例三:毛发引起的爬行疹也被描述为嵌入的头发、刚毛 迁徙者、挖洞的头发、匍匐的头发、迁移的头发和皮毛疹 ${ }^{[6-8]}$ 。皮 肤毛迁移涉及的位置包括脚踝、脚底、脚趾、乳房、脸项或颈 部、下巴和腹部, 而本例患者位于左腿的后侧, 以前未曾有过 报道。在所有被报道的儿童病例中, 足部都发现了这样的毛发 碎片, 表皮破裂可能是毛干进入皮肤的前提, 鞋子和脚之间的 摩擦似乎是一个诱因。导致毛发轴迁移的不是自动移动, 而是 身体表面运动的力量, 就像头发轴自己在爬行。在案例三中, 导致毛发迁移的原因可能是母亲长期抱孩子，母亲掉落的头 发经贞儿伤口处进入组织, 再加上贞儿活泼好动, 导致毛发轴 迁移。

\section{参考文献}

[1]王琪.眼里“奇莳”多养护需到位[J].健康讲堂,2014(9):21-22.

[2]Mahdavi Fard A, Pourafkari L. The Hairy Eyeball-Limbal Dermoid[J]. N Engl J Med, 2013,368(1):64.

[3]Hiu Xie, Ru-zhi Zhang, Wen-yuan Zhu. A new site of cutaneous pili migrans in a 6-month-old infant [J]. Indian J Dermatol Venereol Leprol, 2012,78(4):498-499.

[4]马济宏.虫和䖝传播的疾病[J].家庭医学,1998(9):24.

[5]邭毅,姚军,周薇,等.头䖝在传播乙肝病毒中的作用[J].中国学校 卫生,1995( 4):267-268.

[6]Yaffee HS. Imbedded hair resembling larva migrans [J]. Arch Dermatol, 1957(76):254.

[7]Sakai R, Higashi K, Ohta M, et al. Creeping hair: an isolated hair burrowing in the uppermost dermis resembling larva migrans [J]. Dermatology, 2006(213):242-244.

[8]Kim JY, Silverman RA. Migrating hair: A case confused with cutaneous larva migrans[J]. Pediatr Dermatol, 2010,27(6):628-630. 